\title{
Digital Orthophoto Generation with Aerial Photographs
}

\author{
1Lawali Rabiu \\ 2Dauda Waziri A. \\ $1 \& 2$ Federal Polytechnic Damaturu, Yobe State, Nigeria \\ Email: lawalirabiu@gmail.com
}

Doi:10.5901/ajis.2014.v3n7p133

\begin{abstract}
Orthophoto process transforms a vertical aerial photograph in to the equivalent of traditional map. Yet it retains the advantages of a photograph-virtually displaying actual cultural and land features and the built environment rather than representing those features using symbols and lines. With the development of technology, Digital photogrammetry has started to be used widely in almost all areas about mapping. Especially digital orthophotos which are a photogrammetric products, are being used by lots of private sectors because of theirs easy interpretability. Several photogrammetric software which can be use to generate orthophotos are Leica Photogrammetric System (LPS), PCI Geomatica, Topcon pi 3000, Image Master, Erdas Imagine, VirtuoZo and others. In this study photogrammetric data from aerial photographs was used to generate orthophoto using erdas imagine software. The processing procedure of the system is described in detail. Sets of measurements (length and width) of selected buildings were obtained. The results obtained from this software are compared with ground survey measurement. The results showed that an accuracy of sub-meter of $\pm 0.406 \mathrm{~m}$ could be obtained from orthophoto generated using erdas imagine software. Conclusion is drawn concerning the suitability of the software for use in orthophoto generation. Future research is recommended based on two or more photogrammetric systems to comprise both side overlap and end overlap.
\end{abstract}

Keywords: Digital orthophoto, DTM, aerial photograph, Erdas Imagine,

\section{Introduction}

Digital photogrammetry is photogrammetry applied to digital images that are stored and processed on a computer. Digital images can be scanned from photographs or directly captured by digital cameras. Many photogrammetric tasks can be highly automated in digital photogrammetry (e.g. automatic DEM extraction and digital orthophoto generation). Digital photogrammetry is sometimes called softcopy photogrammetry. The output products are in digital form, such as digital maps, DEMs, and digital orthoimages saved on computer storage media. Therefore, they can be easily stored and managed by the user. With the development of digital photogrammetry, photogrammetric techniques are more closely integrated in to remote sensing and GIS.

Orthophotos are photographs that have been corrected for distortions due to tilting of the camera during the photographic survey, distortions from camera lens, and relief distortions. Orthophotos display all the valuable information of a photograph, but unlike a photograph, true distance, angles, and areas can be measured directly (Rossi, 2004)

Orthophoto imagery is a valuable layer in a GIS because it provides a generic, inexpensive, and accurate base map. However, in cities with tall buildings it is difficult to create a seamless data base of orthophoto imagery. Traditional methods for orthophoto rectification disregard buildings. (Wolfgan S, et al, 1998).

In recent years, many technological advances have been made in the field of photogrammetry, the process of making maps using photographs. Perhaps the most significant achievement has been the refinement of methods to produce digital orthophotographs. Digital orthophotos are scaled aerial photographs, which can be used (among other things) as a base map in a GIS or as a tool to revise digital line graphs and topographic maps (Eric T. 1998).

Besides, up to date and spatial information can be provided by the help of digital photogrammetry using aerial photos and satellite images. The orthophoto maps of digital photogrammetric production, which are produced by using aerial photographs and satellite images can supply fast and economical map production. Especially the orthophoto maps that are produced by using satellite images, by means of developing technology, are advancing for being economical alternatives to classical maps. Even the updating of digital vector products take a short time, at first step the production takes too much time (Akdeniz, 2004).

A very common application of aerial photography is the creation of orthophotos - photographs which have been 
"corrected" so as to be usable as a map. In other words, an orthophoto is a simulation of a photograph taken from an infinite distance, looking straight down. Perspective must obviously be removed, but variations in terrain should also be corrected for. Multiple geometric transformations are applied to the image, depending on the perspective and terrain corrections required on a particular part of the image.

Orthophotos are commonly used in geographic information systems, such as are used by mapping agencies (e.g. Ordnance Survey) to create maps. Once the images have been aligned, or 'registered', with known real-world coordinates, they can be widely deployed.

Large sets of orthophotos, typically derived from multiple sources and divided into "tiles" (each typically 256 x 256 pixels in size), are widely used in online map systems such as Google Maps. Open Street Map offers the use of similar orthophotos for deriving new map data. Google Earth lays orthophotos onto a digital elevation model to simulate 3D landscapes.

An orthophoto, orthophotograph or orthoimage is an aerial photograph geometrically corrected ("orthorectified") such that the scale is uniform: the photo has the same lack of distortion as a map. Unlike an uncorrected aerial photograph, an orthophotograph can be used to measure true distances, because it is an accurate representation of the Earth's surface, having been adjusted for topographic relief, lens distortion and camera tilt.

Orthophotographs are commonly used in the creation of a Geographic Information System (GIS). Software can display the orthophoto and allow an operator to digitize or place line work, text annotations or geographic symbols (such as hospitals, schools, and fire stations). Some software can process orthophoto and produce the line work automatically.

The purpose of this paper is to generate orthophoto using photogrammetric data. The paper also includes detail discussion of the processing procedure and analysis of the results obtained from the measurements made.

\section{Methodology}

\subsection{Input Data}

The two overlapping aerial photographs covering part of Universiti Teknologi Malaysia campus (photo number 67 and photo number 68), camera parameters, and ground control points (GCPs) were used as input data (Figure 1.1). This data were then being processed using Erdas Imagine software. Arial photograph 2001; scale 1:10,000.

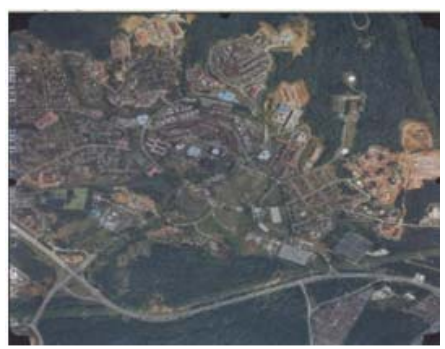

Photo 67

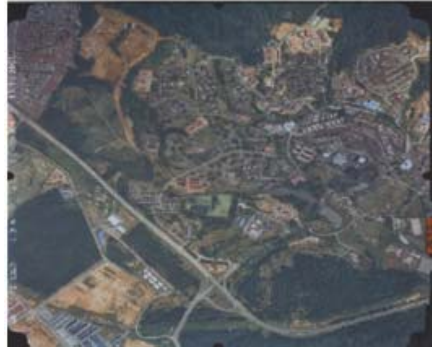

photo 68

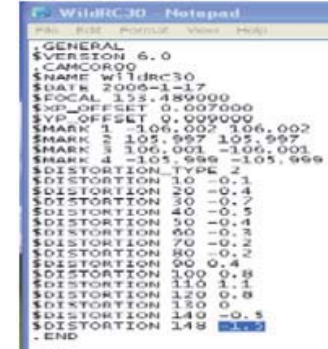

Camera parameters

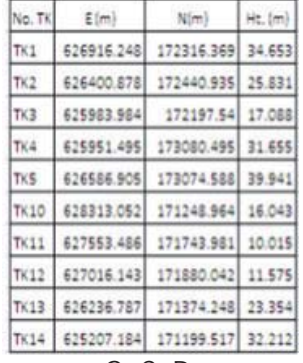

G. C.P.

Figure 1.1: Input Data

Source: Universiti Teknologi Malaysia

\subsection{Data processing}

Digital photogrammetric systems employ sophisticated software to automate the tasks associated with conventional photogrammetry, thereby minimizing the extent of manual interaction required to perform photogrammetric operations. IMAGINE orthoBASE (Figure 1.2) is such a photogrammetric system.

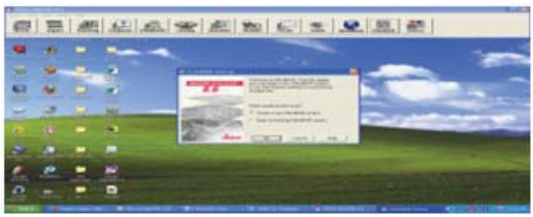

Figure 1.2: Imagine OrthoBAE 


\subsection{Processing Procedure}

The procedures of generating digital orthophoto using Erdas Imagine software are; Creation of Imagine Orthbase project, Creation of Image Pyramid, Interior Orientation, Exterior Orientation, DTM extraction, and Orthorectification.

During the creation of imagine orthobase project the following tasks were conducted; selection of geometrical model, setting reference system, setting reference units, and setting specific information. Figure 1.3 shows imagine orthobase project (block file) created before adding image,

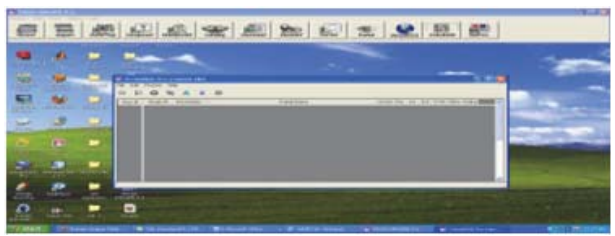

Figure 1.3: block file

\subsubsection{Image Pyramid}

Because of the large amount of image data, the image pyramid is usually adopted during the image matching techniques to reduce the computation time and to increase the matching reliability. The pyramid is a data structure consisting of the square image represented several times, at a decreasing spatial resolution each time. Each level of the pyramid contains the image at a particular resolution. Pyramid of images (photo67 and photo68) was generated; the pyramid column has now turned from red to green as shown in figure 1.4 below.

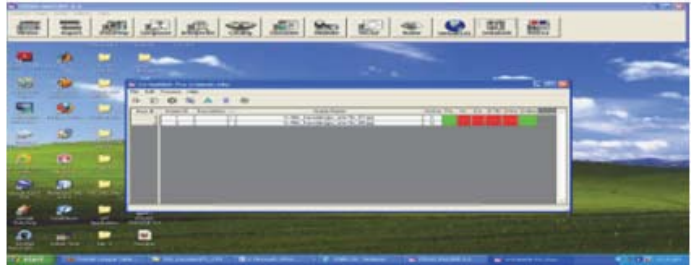

Figure 1.4: pyramid

\subsubsection{Interior Orientation}

Interior orientation defines the internal geometry of a camera or sensor as it existed at the time of image capture. The variables associated with image space are defined during the process of defining interior orientation. Interior orientation is primarily used to transform the image pixel coordinate system or other image coordinate measurement system to the space coordinate system. The camera information were inputted such as general, fiducial, and radial lens distortion, in the frame editor the fiducial marks were digitized, and finally the interior orientation column has now turned from red to green as shown in figure 1.5 below.

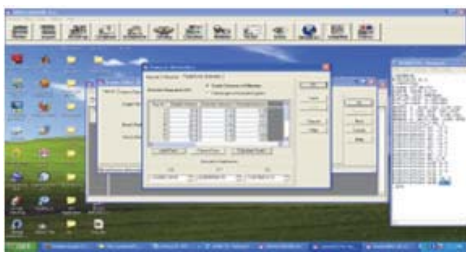

Camera Information

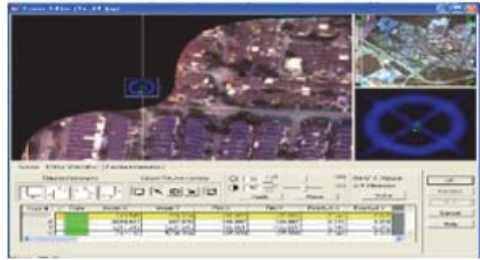

Frame Editor

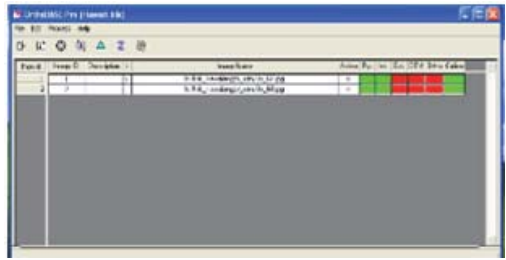

Interior Orien. Completed

Figure 1.5: Interior Orientation

\subsubsection{Exterior Orientation}

Exterior orientation defines the position and angular orientation of the camera that captured an image. The variables 
defining the position and orientation of an image are referred to the as elements of exterior orientation. The elements of exterior orientation define the characteristics associated with an image at the time of exposure or capture. The positional elements of exterior orientation include $X_{0}, Y_{0}$, and $Z_{0}$. They define the position of the perspective centre (0) with respect to the ground space coordinate system (X, $Y$, and $Z)$. Zo is commonly referred to as height of the camera above sea level, which is commonly defined by a datum. Aerial triangulation was carried out with the aid of the established ground control points as shown in figure 1.6 below.

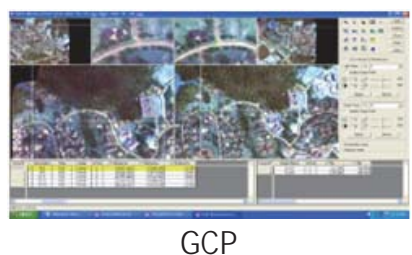

GCP

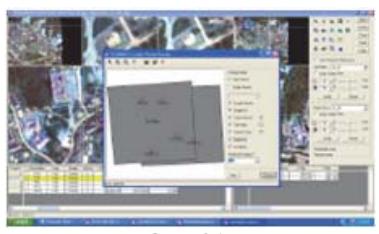

Graphic

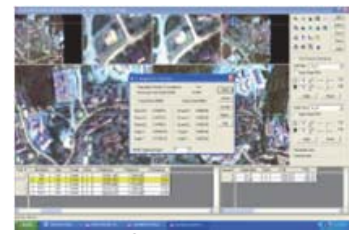

Summary

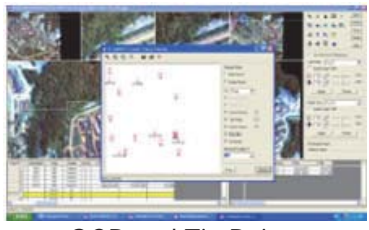

GCP and Tie Points

Figure 1.6: Aerial Triangulation

Five GCPs were used in the overlapping photographs, the graphical status of GCPs and Tie Points with their ID's, and the RMSE of the triangulation summary was found to be \pm 0.3225 . It is an acceptable value to further process the imagery.

\subsubsection{DTM Creation}

Various techniques and approaches can be used for the creation of DTMs. These techniques vary with respect to the technology used to collect elevation information from the Earth's surface: the different techniques include:

- Ground Survey

- Photogrammetry

- Digital stereo plotters

- Digitized topographic maps

- Radar

- Light detection and ranging (LIDAR)

In this project DEM output DTM type and single mosaic were selected, and the DTM file name dtm6768.img, the cell size of 10 meters in both $x$ and $y$ axis with the pixel made square in size, the DTM border was trimmed by $10 \%$ and finally contour line with an interval of 5.00 meters was generated. The DTM and generated contours are shown in figure 1.7 below.
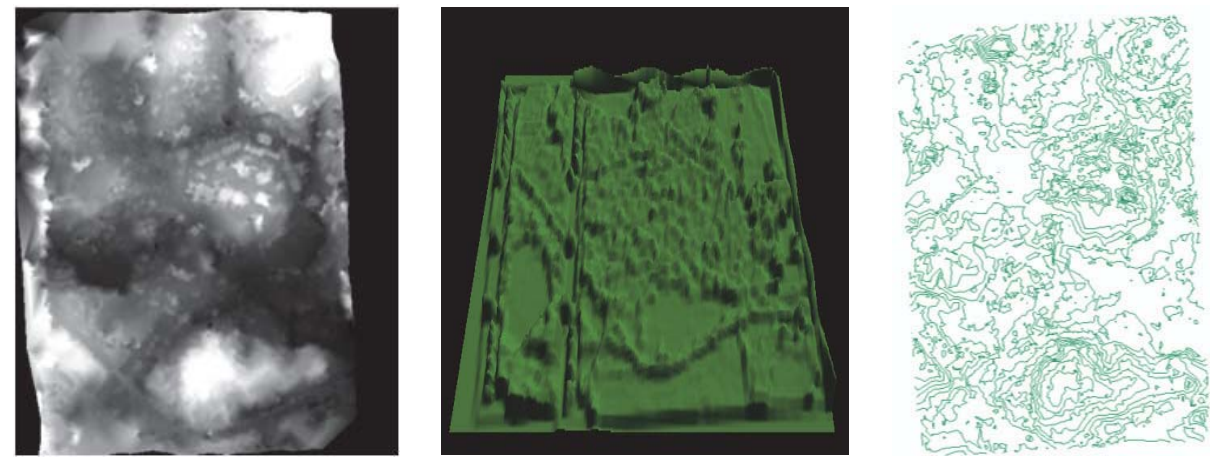

\section{Figure 1.7: DTM}

\subsubsection{Orthorectification}

Orthorectification is the process of reducing geometric errors inherent within photography and imagery. The variables contributing to geometric errors include, but not limited to:

- Camera and sensor orientation 
- Systematic error associated with camera and sensor

- Topographic relief displacement

- Earth curvature

By performing block triangulation or single frame resection, the parameters associated with camera and sensor orientation are defined. Utilizing least squares adjustment techniques during block triangulation minimizes the errors associated with camera and sensor instability. Additionally, the use of self-calibrating bundle adjustment (SCBA) techniques along with interior geometry. The effects of the earth's curvature are significant if a large photo block or satellite imagery is involved. They are accounted for during the block triangulation procedure by setting the proper option. The effects of topographic relief displacement are accounted for by utilizing a DEM during the orthorectification procedure.

Figure 1.8: Workflow

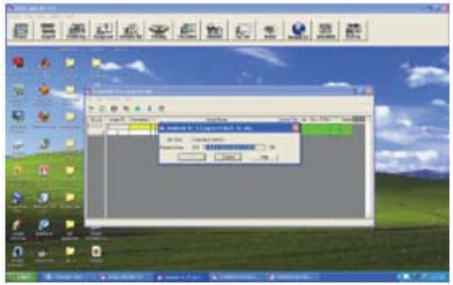

In this study, the process of digital photogrammetric workflow is linear. For instance, in this study where by frame cameras is used, automatic tie point collection cannot begin until fiducial marks have been measured for each image in the block. Using the icons located within the orthoBASE dialog, moving through the various steps from left to right allows for the completion of the photogrammetric workflow, see figure 1.8. Figure 1.9a shows the orthophoto generated in this study. The area (overlap) in between the red and green shaded area is used for further processing. The overlap area (steromodel) of the stereomodel can be used for mapping and analysis, figure $1.9 \mathrm{~b}$.

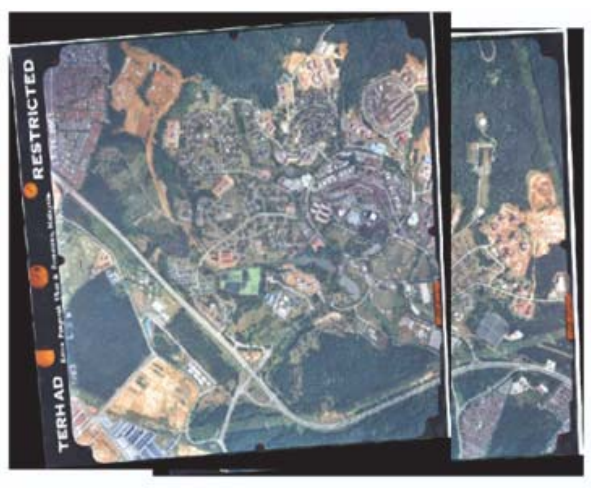

Figure 1.9a: Orthophoto

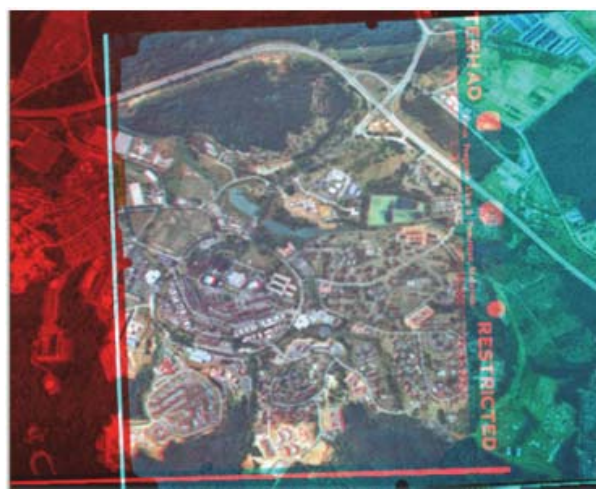

Figure 1.9b: Area of overlap

Figure 1.9: Stereomodel

\subsection{Quality Assessment of the Method}

The results of the measurement made from stereomodel generated using Erdas Imagine software were compared with the results obtained using ground survey method. The measurements made using ground survey method were used as 'reference value' or 'accepted value'. Figure 1.10 shows five building blocks that were used for analysis in this study. Heights and length of each building were measured three times. 


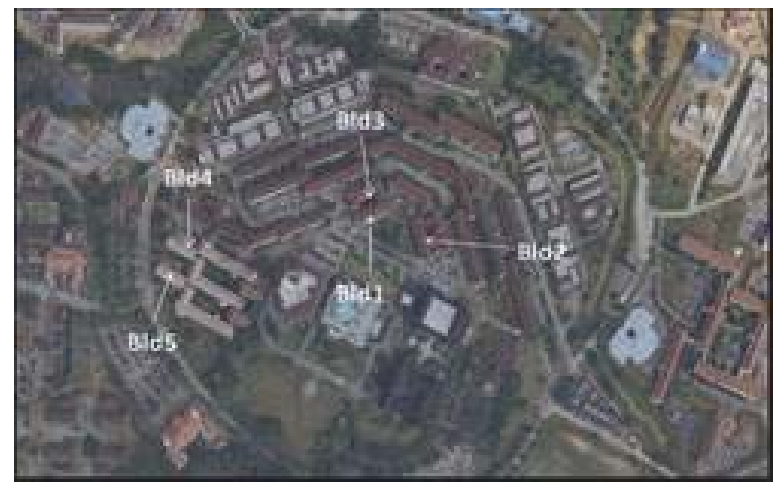

Figure 1.10: Building blocks

In this study, quantitative assessment is used. The Root Mean Square Error (RMSE) is used to determine the accuracy achieved by orthophoto. The lower the value of RMSE the higher is the accuracy and vice-versa.

Table 1 shows the RMSE obtained in this study by comparing the measurement made from the orthophoto and ground survey measurement. It shows the final result of the methods.

Table 1: Final results of all the measurements

\begin{tabular}{|c|c|c|c|c|c|c|}
\hline \multirow{2}{*}{ Building } & \multicolumn{2}{|c|}{ Orthophoto } & \multicolumn{2}{c|}{ Ground Survey } & \multicolumn{2}{c|}{ Differences $(\mathrm{m})$} \\
\cline { 2 - 7 } & Length $(\mathrm{m})$ & Width $(\mathrm{m})$ & Length $(\mathrm{m})$ & Width $(\mathrm{m})$ & Length $(\mathrm{m})$ & Width $(\mathrm{m})$ \\
\hline 1 & 54.803 & 18.441 & 54.646 & 18.649 & 0.157 & -0.208 \\
\hline 2 & 53.944 & 18.718 & 54.532 & 18.334 & -0.588 & 0.384 \\
\hline 3 & 54.043 & 18.439 & 54.107 & 18.251 & -0.064 & 0.188 \\
\hline 4 & 41.842 & 14.251 & 41.526 & 14.912 & 0.316 & -0.661 \\
\hline 5 & 41.24 & 15.697 & 41.033 & 15.303 & 0.207 & 0.394 \\
\hline \multicolumn{3}{|c}{} \\
\hline
\end{tabular}

Table 2: RMSE Error Vector

\begin{tabular}{|c|c|c|}
\hline \multirow{2}{*}{} & \multicolumn{2}{|c|}{ Orthophoto } \\
\cline { 2 - 3 } & Length $(\mathrm{m})$ & Width $(\mathrm{m})$ \\
\hline RMSE $(\mathrm{m})$ & 0.18 & 0.226 \\
\hline Error Vector & 0.406 & \\
\hline
\end{tabular}

The graphical representation of the RMSE in terms of length and width measurements as listed in Table 1 is shown in Figure 1.11.

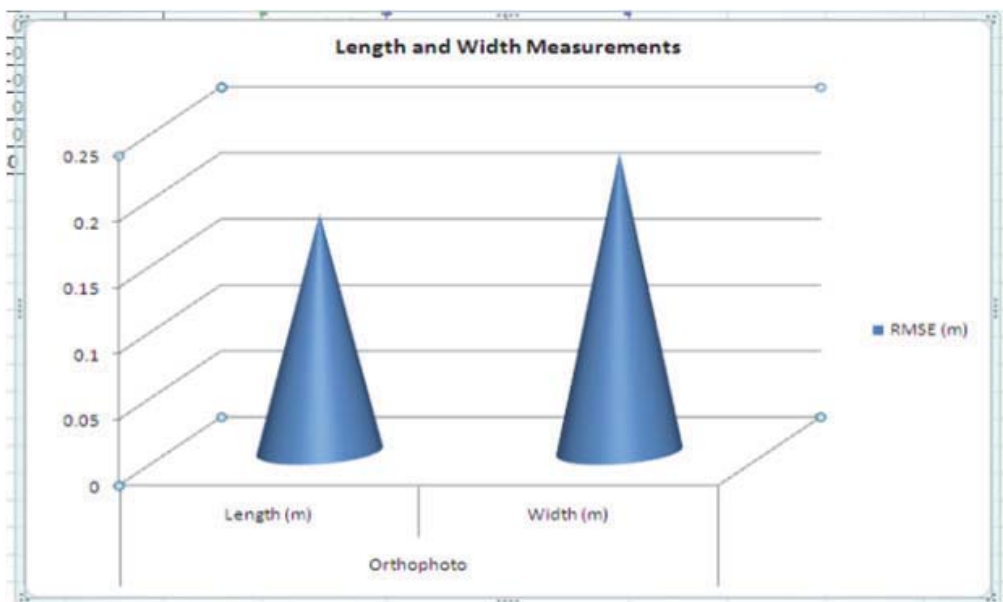

Figure 1.11: RMSE of Length and Width Measurements 
Final result Measurements of all the methods listed in Table 1 is graphically represented in Figure 10.12. It reveals that none of the methods is better than the rest in all ramifications.

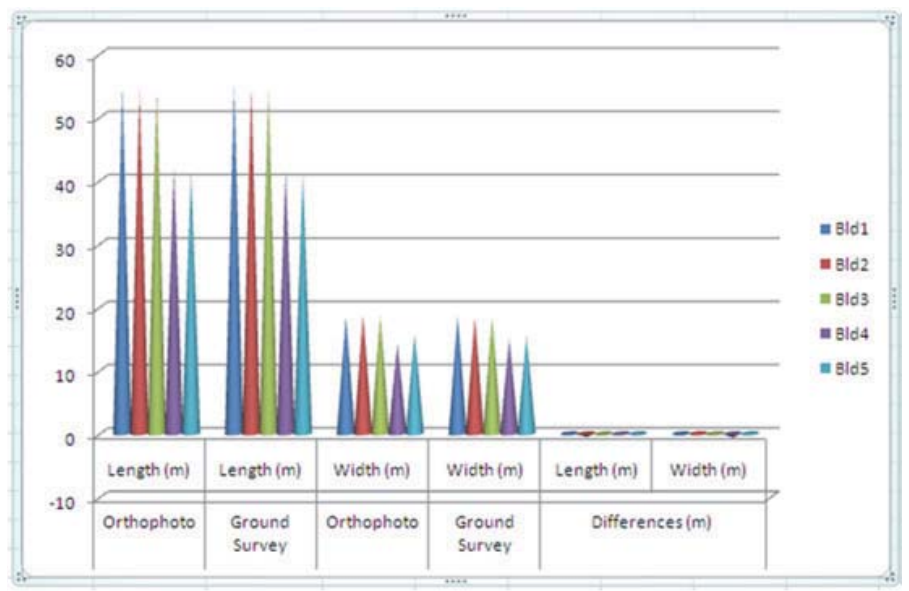

Figure 10.12: Final results (mean) for length and width measurements

In this study, the statistical analysis carried out is similar to work done by Ahmad (1992). In order to compare the standard deviations (or variances) of the methods of measurement used in the study, a statistical testing procedure was carried out. The statistical procedure is known as Variance Ratio Test. In this study the variance ratio test will be used in order to find out whether there is any significant difference in the performance of the method used in the study. This can enable the user to find out whether or not the method came from same populations with the same variance by comparing the variance of any two samples of the methods used. From the outcome of the test, if the two samples come from the same population then they can be considered similar.

For instance, if $\mathrm{s}_{1}$ and $\mathrm{s}_{2}$ are the variance of samples 1 and 2 computed with $\mathrm{v} 1$ and $\mathrm{v} 2$ degree of freedom then the two sided test can be set up as follows:

$H_{0}: \sigma_{1}=\sigma_{2}$

$H_{A}: \sigma_{1} \neq \sigma_{2}$

Where

$\mathrm{Ho}_{\mathrm{o}}$ is the Null Hypothesis that states that the two samples come from population of the same variance.

$\mathrm{H}_{\mathrm{A}}$ is the Alternative Hypothesis that states the two samples come from population of different variance. $\sigma_{1}$ and $\sigma_{2}$ are the (unknown) variance of the two populations.

The $\mathrm{F}$ (Fisher) Distribution which is the ratio of the squares of variance of the samples is to be determined in the next step as follows:

$\mathrm{F}=\mathrm{S}_{1}{ }^{2} / \mathrm{S}_{2}{ }^{2}$

Where;

s1>s2 and has a F distribution with $\mathrm{v}_{1}$ and $\mathrm{V}_{2}$ degree of freedom

For this study the degree of freedom is two (i.e. v1=v2=10). A suitable level of significant set for the two sided test is 0.1 (i.e. 10\%), this means that the probability of accepting the Null Hypothesis is $90 \%$. The F distribution at significant level $a=0.05$, with $v 1=10$ and $v 2=10$ from the Percentile table is 2.99 . The next stage is to compute the values of the $F$ statistics. Ground Survey method is considered to have the smallest error vector $(0.000)$ and hence this value has been taken as $s_{2}$ and orthophoto measurement is taken as $S_{1}$ in turn. As an illustration to compute the $F$ statistics between Ground survey (i.e. $s_{2}=0.000$ ) and orthophoto (i.e. $s_{1}=0.406$ ), we have the solution as:

$F=(0.000)^{2} /(0.406)^{2}=0.000$

The computed $F$ statistics results are listed in Table 5.13, this values are compared with the $F$ statistics value from the Percentile Table and the results of the two sided are given (either accepted or rejected).

Table 2: Results of the Variance Ratio Test

\begin{tabular}{|c|c|c|c|}
\hline & F Statistics & Table Value & Null Hypothesis \\
\hline Orthophoto & 0 & 2.99 & Accepted \\
\hline
\end{tabular}


The Null Hypothesis is accepted as the computed values of the F (Fisher) statistics is less than the Tabulated value obtained from the Percentile Table. However, it can be concluded that there is no significant difference between Orthophoto and Ground measurements. The F statistics values for falls within the tolerance value (2.99). It shows that it fall within the same population with ground measurement. The F statistics value for is much closer to the tolerance value (2-99) The overall result reveals that orthophoto can be used in order to achieve the same accuracy with ground measurement in large scale mapping.

\section{Conclusions}

The technological advancement of photogrammetry is software and computer based, just like with all new things. It is absolutely necessary to assess the capabilities and suitability of its application to guide the users and also act as a motivation to the developers themselves to further improve on the aspects of their respective software where it deem to be necessary.

In this study, orthophoto was successfully generated from photogrammetric data using Erdas IMAGINE Software. The most important feature of image based measurement is the fact that none of the objects are being touched during measurement. However, with the emergent of modern technology such as softcopy photogrammetry or digital photogrammetry, this non-contact measurement can be used to produce the orthophoto. .

Furthermore, analysis of the method used in this research can be done based on ground survey. The results revealed that none of the method is better in all ramifications (height and width measurement) than the others. The Null hypothesis was accepted as the computed value of the $\mathrm{F}$ statistics was less than the value obtained from the Percentile Table. It can be concluded that there is no significant difference between these systems. This shows that they are from the same population. In the variance ratio test the measurements fall within the tolerance value. They can be used in order to achieve same accuracy.

The RMSE values in terms of length and width measurements made from orthophoto are 0.180 and 0.226 respectively (Table 5.8). It can be concluded that orthophoto generated with aerial photos using Erdas Imagine software should be used, in order to achieve accurate mapping.

\section{Recommendation}

As a way of improving beyond the scope of this study, I would like to recommend that future research be done in this area "Generating orthophoto using photogrammetric data" based on two or more photogrammetric systems such as PCl Geomatica, DVP, and Leica Photogrammetric system (LPS). So that comparism can be made with ground survey measurement to determine which one is better for a preference to be made.

This research was carried out using two photographs (photo 67 and photo 68), for more study and understanding, I do recommend that future research to be carried out in the same manner, however it should be expanded further by using four or more photographs so that it will comprise both the side overlap and end overlap.

In field surveying in difficult areas, such as marshy, extreme slopes, hardship, hazardous rock formations etc. orthophoto is recommended for accurate mapping as access to much of the property within the project area is not required and most of the work is done under laboratory condition, thus minimizing delays and hardships due to adverse weather condition.

\section{References}

Ahmad A. (1992). An investigation of low cost Photogrammetric Systems using Small Format Photography for use in the recording of buildings. Postgraduate Dissertation, Department of Surveying, University of Newcastle upon Tyne.

Akdeniz, H., The Opportunities That Digital Orthophotos Can Supply In Urban Planning, XXth ISPRS Congress, $12-23$ July 2004, Istanbul, PS WG VII/4, http://www.isprs.org/istanbul2004/comm7/papers/112.pdf,

Alexandrov, A., Hristova, T., Ivanova, K., Koeva, M., Madzharova, T. and Petrova V., H., Aplication Of Quickbird Satellite Imagery For Updating Cadastral Information, Xth ISPRS Congress, 12-23 July 2004, Istanbul, Commission II, http://www.isprs.org/istanbul2004/comm2/papers/160.pdf

Frere, D., Vandekerckhove, J., Moons, T., and Van Gool, L. (1998). Automatic modelling and 3D reconstruction of urban buildings from aerial imagery. In IEEE International Geoscience and Remote Sensing Symposium Proceedings, Seattle, pp.2593-2596.

Ildiko, S., and George, V. (2004). Reconstruction of 3D building models from aerial images and maps. ISPRS Journal of Photogrammetry \& Remote Sensing 58 (2004) 202-224.

Narushige, S. (2001). 3D Urban Models: Recent developments in the digital modeling of urban environments in three-dimensions. 
Geojournal 52: 263-269, 2001. @ 2001 Kluwer Academic Publishers. Printed in Netherlands.

Pedro, M., Jose, L., Alvaro, M., Jaime, G. and Eduardo, Z. (2009). A practical approach to making accurate 3D layouts of interesting cultural heritage sites through digital models. Journal of Cultural Heritage (2009), doi:10.1016/j.culher.2009.02.007.

P.R. Wolf and C.D. Ghilani, 2006. Elementary Surveying: An Introduction to Geomatics, Eleventh edition. Pearson Prentice, Pearson Education, inc.

Remondino, F. and El-Hakim, S.F. (2006). Image-based 3D modeling: a review, Photogrammeric Rec. J. 21 (115) (2006) $269-291$.

Rossi, Tiffiny Ann, Application Of Digital Photogrammetric Methods In The Of Land Cover Change On The Coastal Dunes Of Warren Dunes State Park, Berrien Country, Master Thesis, The Michigan State University, Michigan, 2004.

Taylor and Francis inc. New York, NY 10001. Wilfred, L., 2003. Digital Photogrammetry: Theory and Applications, Spring-Verlag, Berlin Heideburg 2003. Printed in Germany.

Wong, S. S., and Chan, K. L.(2009). 3D object model reconstruction from image sequence based on photometric consistency in volume space. Received: 8 July 2008 / Accepted: 16 July 2009_Springer- Verlag London Limited 2009. 